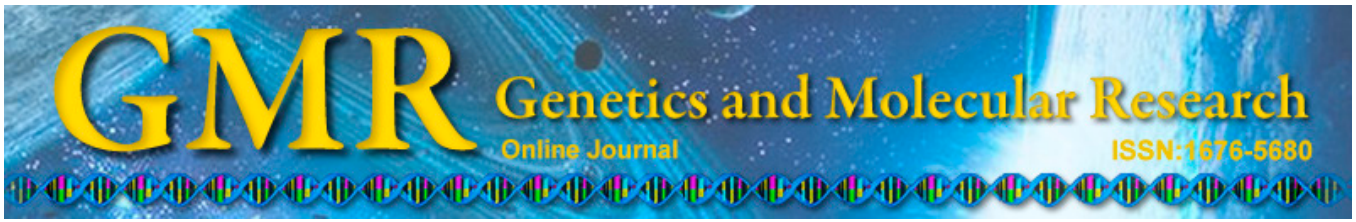

\title{
Role of TGFB1 polymorphism in the development of metastatic brain tumors in non-small cell lung cancer patients
}

\author{
H.B. Wang, W.G. Song, H.Q. Liu, F. Fang and Y. Xiao \\ Department of Radiotherapy and Chemotherapy Tangshan Gongren Hospital, \\ Tangshan, China \\ Corresponding author: H.B. Wang \\ E-mail: haibowang1980@yeah.net
}

Genet. Mol. Res. 14 (2): 3545-3550 (2015)

Received March 25, 2014

Accepted August 22, 2014

Published April 17, 2015

DOI http://dx.doi.org/10.4238/2015.April.17.3

\begin{abstract}
We conducted a case-control study to investigate the role of 3 single-nucleotide polymorphisms of the gene encoding transforming growth factor- $\beta 1$ (TGFB 1) in the development of metastatic brain tumors in non-small cell lung cancer patients. The polymorphisms in TGFB1 rs 4803455 , rs 1800469 , and rs1800470 were evaluated by polymerase chain reaction-restriction fragment length polymorphism. Odds ratios and their corresponding 95\% confidence intervals were used to assess the influence of TGFB1 rs 4803455 , rs1800469, and rs1800470 on metastatic brain tumors. We found that cases were more likely to have a later disease stage when compared with control subjects, without brain metastasis. Individuals carrying the TGFB1 rs1800469 TT and CT+TT genotypes had an increased risk of developing brain metastasis compared with the rs $1800469 \mathrm{CC}$ genotype. Moreover, a significant interaction was observed between the rs 1800469 polymorphism and disease stage. However, no significant association between polymorphisms rs 4803455 and rs 1800470 and the risk of developing brain metastasis were observed. We found that the TGFB1 rs1800469 polymorphism may be predictive biomarker for the risk of developing brain metastasis in non-small cell
\end{abstract}


lung cancer patients.

Key words: Brain metastasis; Non-small cell lung cancer; Polymorphism; TGFB1

\section{INTRODUCTION}

An estimated 150,000 patients are diagnosed with brain metastases every year, and non-small cell lung cancer (NSCLC) is the most common primary site for secondary brain metastasis (Subramanian et al., 2002; Nathoo et al., 2005; Bovi and White, 2012). With advanced therapy and increased use of combined-modality therapy, the distant metastases of lung cancer have been reduced and overall survival has improved. However, overall brain failure is increasing (Gore et al., 2011). Even in patients with good performance status and controlled extra-cranial disease, the median survival time is approximately 7 months (Gaspar et al., 2000). Therefore, identifying patients at risk of developing metastatic brain tumors can lead to more effective prevention measures for these patients.

Transforming growth factor- $\beta$ (TGF- $\beta$ ) is one type of TGF, and signaling pathways of TGF- $\beta$ can influence cell proliferation, morphogenesis, migration, extracellular matrix production, and apoptosis. Previous studies have shown that TGF- $\beta$ can suppress early-stage tumor development through its potent growth inhibitory effects, but that it is a pro-oncogenic factor that stimulates tumor cell growth and invasiveness during end-stage tumorigenesis (Javelaud et al., 2008; Massagué, 2008). Tumor cells can escape the antiprolifeative action of TGF- $\beta$ by developing mutations in components of relevant signaling pathways or by disrupting TGF- $\beta$ signaling. Therefore, TGF- $\beta$ expression may play a role in the metastatic process.

In our study, we examined whether common genetic polymorphisms of TGF- $\beta$ pathways play a role in maintaining cellular function and their effect on distant metastasis. We conducted a case-control study to investigate the role of 3 single-nucleotide polymorphisms (SNPs) of TGFB1 in the development of metastatic brain tumors in NSCLC patients.

\section{MATERIAL AND METHODS}

\section{Patient population}

A total of 176 subjects in this study were selected from Tangshan Gongren Hospital between January 2007 and December 2009. All cases with NSCLC were pathologically diagnosed. All patients had at least a single brain metastasis of NSCLC and subsequently underwent either brain radiotherapy or local brain radiotherapy. A total of 198 patients with NSCLC and without brain metastasis were included as a control group. Patients with any other malignant tumor history within the past 5 years, pregnancy or lactation, cardiopulmonary insufficiency, serious cardiovascular disease, and serious infection as well as severe malnutrition were excluded from the study.

Demographic and clinical data were obtained from medical records, including gender, age, disease stage, tumor histology, and smoking and drinking habits. The disease stage of patients was classified based on the tumor/node/metastasis (TNM) system according to the American Joint Committee on Cancer staging manual. The diagnosis of brain metastasis was conducted based on computed tomography and magnetic resonance imaging records. Smok- 
ing habit was classified into never and ever smokers. Individuals who smoked 20-50 packs of cigarettes per year, or smoked more than 1 cigarette per day continuously for 6 months were regarded as smokers. The drinking habit was classified as never and ever drinkers. Subjects who drank more than $50 \mathrm{~g}$ alcohol per week continuously for 6 months were regarded as drinkers.

\section{Genotype analysis}

Each case and control subject provided $5 \mathrm{~mL}$ venous blood. Ethylenediaminetetraacetic acid $(1.5-2.2 \mathrm{mg} / \mathrm{mL})$ was used as an anticoagulant during blood sample storage. DNA was extracted using the TIANamp blood DNA kit (Tiangen Biotech; Beijing, China) according to manufacturer instructions. The TGFB1 polymorphisms rs4803455, rs1800469, and rs 1800470 were analyzed by polymerase chain reaction (PCR)-restriction fragment length polymorphism. The primers used for TGFB1 rs 4803455 , rs 1800469 , and rs 1800470 were designed using the Sequenom Assay Design 3.1 software (San Diego, CA, USA). Each PCR mix was conducted in a total volume of $20 \mu \mathrm{L}$ and included $50 \mathrm{ng}$ genomic DNA, $200 \mu \mathrm{M}$ dNTPs, and $2.5 \mathrm{U}$ Taq DNA polymerase as well as $200 \mu \mathrm{M}$ primers. The PCR amplification was started with preliminary denaturation at $95^{\circ} \mathrm{C}$ for $10 \mathrm{~min}$ to activate the Taq polymerase, and consisted of 45 cycles $\left(95^{\circ} \mathrm{C}\right.$ for $5 \mathrm{~s}$ and $60^{\circ} \mathrm{C}$ for $\left.30 \mathrm{~s}\right)$, with a final extension at $72^{\circ} \mathrm{C}$ for $3 \mathrm{~min}$.

\section{Statistical analysis}

All statistical analyses were conducted using SPSS version 11.0 (SPSS Inc., Chicago, IL, USA) for Windows. Continuous variables are expressed as means $\pm \mathrm{SD}$, while categorical variables are expressed as $\mathrm{N}$ of subjects (\%). Continuous variables and categorical variables were analyzed using the independent sample $t$-test or $\chi^{2}$-test, respectively. Odds ratios (ORs) and their corresponding 95\% confidence intervals (CIs) were used to assess the influence of TGFB1 rs4803455, rs1800469, and rs 1800470 on metastatic brain tumors. The homozygous genotypes for the 3 SNPs were used as references. All comparisons were 2-sided, and $\mathrm{P}<0.05$ was considered to be statistically significant.

\section{RESULTS}

A total of 176 cases and 198 control subjects were enrolled in our study. The general characteristics of all subjects are summarized in Table 1. No significant difference was found between cases and controls in terms of gender, age, smoking, and drinking habits $(\mathrm{P}>0.05)$. Cases were more likely to have a higher disease stage compared with control subjects.

In the conditional regression analysis, we found that individuals carrying the TGFB1 rs 1800469 TT genotype had an increased risk of developing brain metastasis compared with the rs1800469 CC genotype, with adjusted ORs (95\%CI) of 1.76 (1.12-3.15) (Table 2). Similarly, we found that subjects carrying the rs1800469 CT+TT genotypes had a slightly increased risk of developing brain metastasis, and the adjusted OR $(95 \% \mathrm{CI})$ was $1.46(95 \% \mathrm{CI}$ $=1.03-2.53$ ) after adjusting for gender, age, smoking and drinking habit, and disease stage. However, no significant association between polymorphisms in rs4803455 and rs1800470 and the risk of developing brain metastasis was observed.

We further analyzed the interaction between the TGFB1 rs1800469 polymorphism and disease stage on the risk of developing brain metastasis (Table 3). Individuals with rs 1800469 
TT showed a significant association with a greatly increased risk of developing brain metastasis among patients with disease stage at IIIA-IV $(\mathrm{OR}=2.58,95 \% \mathrm{CI}=1.19-5.67)$. Subjects carrying the rs $1800469 \mathrm{CT}+\mathrm{TT}$ genotypes were associated with a moderately increased risk of developing brain metastasis. Moreover, a significant interaction was found between the rs 1800469 polymorphism and disease stage $(\mathrm{P}=0.03)$.

Table 1. Demographic and clinical characteristics of cases and controls.

\begin{tabular}{|c|c|c|c|c|c|c|}
\hline Characteristics & Patients & $\%$ & Control & $\%$ & $\chi^{2}$ & $P$ value \\
\hline \multicolumn{7}{|l|}{ Gender } \\
\hline Male & 45 & 25.57 & 57 & 28.79 & & \\
\hline Female & 131 & 74.43 & 141 & 71.21 & 0.49 & 0.48 \\
\hline \multicolumn{7}{|l|}{ Age } \\
\hline$<60$ & 73 & 41.48 & 86 & 43.43 & & \\
\hline$\geq 60$ & 103 & 58.52 & 112 & 56.57 & 0.15 & 0.71 \\
\hline \multicolumn{7}{|l|}{ Smoking habit } \\
\hline No & 80 & 45.45 & 96 & 48.48 & & \\
\hline Yes & 96 & 54.55 & 102 & 51.52 & 0.34 & 0.56 \\
\hline \multicolumn{7}{|l|}{ Drinking habit } \\
\hline No & 107 & 60.80 & 126 & 63.64 & & \\
\hline Yes & 69 & 39.20 & 73 & 36.87 & 0.25 & 0.62 \\
\hline \multicolumn{7}{|c|}{ Disease stage at diagnosis } \\
\hline I-II & 84 & 47.73 & 115 & 58.08 & & \\
\hline IIIA-IV & 92 & 52.27 & 83 & 41.92 & 4.01 & 0.05 \\
\hline
\end{tabular}

Table 2. Association between $T G F B 1$ genotypes and brain metastasis.

\begin{tabular}{|c|c|c|c|c|c|c|c|c|}
\hline TGFB1 & Case & $\%$ & Control & $\%$ & OR $(95 \% \mathrm{CI})^{1}$ & $P$ value & OR $(95 \% \mathrm{CI})^{2}$ & P value \\
\hline \multicolumn{9}{|l|}{ rs4803455 } \\
\hline AA & 75 & 42.5 & 90 & 45.3 & 1.0 (Ref.) & - & 1.0 (Ref.) & - \\
\hline $\mathrm{AC}$ & 67 & 37.9 & 74 & 37.5 & $1.09(0.68-1.75)$ & 0.72 & $1.27(0.74-1.95)$ & 0.53 \\
\hline $\mathrm{CC}$ & 34 & 19.6 & 34 & 17.2 & $1.2(0.65-2.20)$ & 0.53 & $1.32(0.81-2.45)$ & 0.43 \\
\hline $\mathrm{AC}+\mathrm{CC}$ & 101 & 57.5 & 108 & 54.7 & $1.12(0.73-1.73)$ & 0.58 & $1.26(0.91-2.05)$ & 0.26 \\
\hline \multicolumn{9}{|l|}{ rs1800469 } \\
\hline $\mathrm{CC}$ & 69 & 39.1 & 92 & 46.3 & 1.0 (Ref.) & - & 1.0 (Ref.) & - \\
\hline $\mathrm{CT}$ & 59 & 33.7 & 64 & 32.5 & $1.23(0.75-2.02)$ & 0.39 & $1.36(0.89-2.27)$ & 0.21 \\
\hline $\mathrm{TT}$ & 48 & 27.2 & 42 & 21.2 & $1.53(0.89-2.65)$ & 0.11 & $1.76(1.12-3.15)$ & 0.04 \\
\hline $\mathrm{CT}+\mathrm{TT}$ & 107 & 60.9 & 106 & 53.7 & $1.35(0.87-2.08)$ & 0.16 & $1.46(1.03-2.53)$ & 0.09 \\
\hline \multicolumn{9}{|l|}{ rs 1800470} \\
\hline $\mathrm{CC}$ & 68 & 38.5 & 84 & 42.3 & 1.0 (Ref.) & - & 1.0 (Ref.) & - \\
\hline $\mathrm{CT}$ & 73 & 41.2 & 80 & 40.4 & $1.13(0.70-1.81)$ & 0.60 & $1.37(0.87-2.02)$ & 0.27 \\
\hline $\mathrm{TT}$ & 36 & 20.3 & 34 & 17.3 & $1.31(0.71-2.40)$ & 0.35 & $1.47(0.84-2.83)$ & 0.15 \\
\hline $\mathrm{CT}+\mathrm{TT}$ & 109 & 61.5 & 114 & 57.7 & $1.18(0.76-1.83)$ & 0.43 & $1.26(0.84-2.07)$ & 0.26 \\
\hline
\end{tabular}

${ }^{1}$ Not adjusted. ${ }^{2}$ Adjusted for gender, age, smoking and drinking habit and disease stage.

Table 3. Interaction between $T G F B 1$ rs1800469 polymorphism and disease stage on the risk of developing brain metastasis.

\begin{tabular}{|c|c|c|c|c|c|c|c|c|}
\hline \multirow[t]{2}{*}{ TGFBI } & \multicolumn{2}{|c|}{ I-II } & \multirow[t]{2}{*}{ OR $(95 \% \mathrm{CI})^{1}$} & \multirow[t]{2}{*}{$P$ value } & \multicolumn{2}{|c|}{ IIIA-IV } & \multirow[t]{2}{*}{ OR $(95 \% \mathrm{CI})^{1}$} & \multirow[t]{2}{*}{$\mathrm{P}$ value } \\
\hline & Case & Control & & & Case & $\overline{\text { Control }}$ & & \\
\hline \multicolumn{9}{|l|}{ rs 1800469} \\
\hline $\mathrm{CC}$ & 36 & 53 & 1.0 (Ref.) & - & 33 & 39 & 1.0 (Ref.) & - \\
\hline $\mathrm{CT}$ & 35 & 38 & $1.52(0.74-2.78)$ & 0.25 & 24 & 26 & $1.17(0.62-2.55)$ & 0.56 \\
\hline $\mathrm{TT}$ & 14 & 24 & $1.06(0.46-2.36)$ & 0.70 & 34 & 18 & $2.58(1.19-5.67)$ & 0.01 \\
\hline $\mathrm{CT}+\mathrm{TT}$ & 49 & 62 & $1.24(0.71-2.15)$ & 0.62 & 58 & 44 & $1.73(1.02-3.46)$ & 0.03 \\
\hline
\end{tabular}

${ }^{1}$ Not adjusted. 


\section{DISCUSSION}

Characterization and identification of genes involved in the genetic predisposition or progression of cancer are critical for both clinical practice and basic medical research. Because the expression of $T G F B 1$ may have a role in the metastatic process, numerous studies have shown that $T G F B 1$ polymorphisms were associated with the prognosis and metastasis of various cancers such as breast, lung, oral, and rectal cancers (Gaur et al., 2011; Slattery et al., 2011; Yuan et al., 2013; Wendt et al., 2014). Only 2 studies reported an association between TGFB1 polymorphisms and risk of brain metastasis (Li et al., 2012; Yuan et al., 2013). However, the results were inconsistent. Therefore, we investigated the influence of TGFB1 polymorphisms on the risk of developing metastasis.

In the present study, we found that the TGFB1 rs1800469 polymorphism was associated with the risk of developing brain metastasis in Chinese NSCLC patients. However, the $T G F B 1$ rs4803455 and rs 1800470 polymorphisms were not associated with the risk of brain metastasis. In addition, in stratified analyses based on disease stage, a significant association was found between the TGFB1 rs 1800469 polymorphism and disease stage.

It is well-known that members of the TGF- $\beta$ cytokine superfamily showed a diverse range of cellular responses, such as proliferation, migration, fibrosis, apoptosis, tissue inflammation, wound repair, and angiogenesis (Massagué, 1998; Roberts, 1999; Massagué and Gomis, 2006). Changes in TGF- $\beta$ signaling have been reported to have an effect on the prognosis of NSCLC (Kang et al., 2000; Finger et al., 2008). For TGF- $\beta$ isoforms, TGFB1 signaling downregulates the migration of tumor cells and suppresses the development of metastasis (Saunier and Akhurst, 2006; Tsuchida et al., 2006). Thus, TGFB1 may play a crucial role in cancer development and the suppression of tumor growth during the initial phases of carcinogenesis, while it plays a role in promoting tumor progression and metastasis during more advanced stages (Derynck et al., 2001; Wakefield and Roberts, 2002). TGFB1 polymorphisms have been used as predictive biomarkers in several cancers (Gaur et al., 2011; Slattery et al., 2011; Li et al., 2012; Yuan et al., 2013; Wendt et al., 2014). Yuan et al. (2013) conducted a study in China and found that TGFB1 genotypes at rs1800469 and rs1982073 can be used as predictive markers for the effectiveness of chemotherapy and prognosis of NSCLC. Gaur et al. (2011) reported that the TGFB1 functional genetic polymorphism at $869 \mathrm{~T}>\mathrm{C}$ was associated with risk of oral cancer in Asian Indians. For brain metastasis in patients with NSCLC, Li et al. (2012) reported a study conducted in China and found no significant association between TGFB1 polymorphisms and brain metastasis. The discrepancy in these results may be explained by the differences in ethnicities, source of control subjects, and sample sizes. Further studies are needed to confirm the association between $T G F B 1$ polymorphisms and risk of brain metastasis.

In conclusion, we found that the TGFB1 rs1800469 polymorphism was statistically associated with the risk of developing brain metastasis in NSCLC patients, and has an interaction with disease stage. However, no association was found between polymorphisms in rs4803455 and rs 1800470 and the risk of brain metastasis. The rs4803455 and rs 1800470 polymorphisms may be used as predictive markers for the development of brain metastasis. A larger sample study is necessary to confirm the role of $T G F B 1$ polymorphisms in the development of brain metastasis. 


\section{REFERENCES}

Bovi JA and White J (2012). Radiation therapy in the prevention of brain metastases. Curr. Oncol. Rep. 14: 55-62.

Derynck R, Akhurst RJ and Balmain A (2001). TGF-beta signaling in tumor suppression and cancer progression. Nat. Genet. 29: 117-129.

Finger EC, Turley RS, Dong M, How T, et al. (2008). TbetaRIII suppresses non-small cell lung cancer invasiveness and tumorigenicity. Carcinogenesis 29: 528-535.

Gaspar LE, Scott C, Murray K and Curran W (2000). Validation of the RTOG recursive partitioning analysis (RPA) classification for brain metastases. Int. J. Radiat. Oncol. Biol. Phys. 47: 1001-1006.

Gaur P, Mittal M, Mohanti BK and Das SN (2011). Functional genetic variants of TGF- $\beta 1$ and risk of tobacco-related oral carcinoma in high-risk Asian Indians. Oral Oncol. 47: 1117-1121.

Gore EM, Bae K, Wong SJ, Sun A, et al. (2011). Phase III comparison of prophylactic cranial irradiation versus observation in patients with locally advanced non-small-cell lung cancer: primary analysis of radiation therapy oncology group study RTOG 0214. J. Clin. Oncol. 29: 272-278.

Javelaud D, Alexaki VI and Mauviel A (2008). Transforming growth factor-beta in cutaneous melanoma. Pigment Cell Melanoma Res. 21: 123-132.

Kang Y, Prentice MA, Mariano JM, Davarya S, et al. (2000). Transforming growth factor-beta 1 and its receptors in human lung cancer and mouse lung carcinogenesis. Exp. Lung Res. 26: 685-707.

$\mathrm{Li}$ Q, Wu H, Chen B, Hu G, et al. (2012). SNPs in the TGF- $\beta$ signaling pathway are associated with increased risk of brain metastasis in patients with non-small-cell lung cancer. PLoS One 7: e51713.

Massagué J (1998). TGF-beta signal transduction. Annu. Rev. Biochem. 67: 753-791.

Massagué J (2008). TGFbeta in cancer. Cell 134: 215-230.

Massagué J and Gomis RR (2006). The logic of TGFbeta signaling. FEBS Lett. 580: 2811-2820.

Nathoo N, Chahlavi A, Barnett GH and Toms SA (2005). Pathobiology of brain metastases. J. Clin. Pathol. 58: 237-242.

Roberts AB (1999). TGF-beta signaling from receptors to the nucleus. Microbes Infect. 1: 1265-1273.

Saunier EF and Akhurst RJ (2006). TGF beta inhibition for cancer therapy. Curr. Cancer Drug Targets 6: 565-578.

Slattery ML, Lundgreen A, Herrick JS, Wolff RK, et al. (2011). Genetic variation in the transforming growth factor- $\beta$ signaling pathway and survival after diagnosis with colon and rectal cancer. Cancer 117: 4175-4183.

Subramanian A, Harris A, Piggott K, Shieff C, et al. (2002). Metastasis to and from the central nervous system - the 'relatively protected site'. Lancet Oncol. 3: 498-507.

Tsuchida K, Sunada Y, Noji S, Murakami T, et al. (2006). Inhibitors of the TGF-beta superfamily and their clinical applications. Mini Rev. Med. Chem. 6: 1255-1261.

Wakefield LM and Roberts AB (2002). TGF-beta signaling: positive and negative effects on tumorigenesis. Curr. Opin. Genet. Dev. 12: 22-29.

Wendt MK, Taylor MA, Schiemann BJ, Sossey-Alaoui K, et al. (2014). Fibroblast growth factor receptor splice variants are stable markers of oncogenic transforming growth factor $\beta 1$ signaling in metastatic breast cancers. Breast Cancer Res. 16: R24.

Yuan X, Wei Q, Komaki R, Liu Z, et al. (2013). TGF $\beta 1$ polymorphisms predict distant metastasis-free survival in patients with inoperable non-small-cell lung cancer after definitive radiotherapy. PLoS One 8: e65659. 